of $\geq 2$ medical comorbidities were significant possible predictors for the development of psychiatric disorders among the included sample (see Table S4, available as supplementary material attached to the electronic version of this paper at www.journals.cambridge.org/jid_IPG).

In this study the rates of current Axis-I DSMIV disorders using the SCID were found to be common, affecting nearly one-third of the sample $(32.6 \%)$, which is in agreement with previous studies (Lyness et al., 1999). However, anxiety disorders appears to be much higher suggesting the need for further rigorous research to identify the factors behind this observation.

Subjects with dementia (29.6\%) were higher than the ratio previously reported among older Saudis by Ogunniyi et al. (1998) (2.1\%). The higher prevalence may be because a larger proportion of the patients with organic mental disorders are being managed at the general hospital set up (i.e. King Fahd Hospital) and continued to follow-up in the outpatient clinics. A clear predominance of males was observed contrary to the findings of similar studies ((Lyness et al., 1999). Women may not attend hospitals as frequently as men for cultural reasons, as they are totally dependent on men to take them there.

Several limitations of this investigation should be recognized. First, since the data is from a hospital situation, the findings cannot extend into the community. Hence the findings need to be confirmed by community-based studies. Second, inferences of causality cannot be accomplished because of the design of this study (cross-sectional). Finally, there was no data of the SCID diagnosis in community or primary care settings in Saudi Arabia for comparison.

In conclusion, psychiatric disorders are common among older persons attending a tertiary care setting in Saudi Arabia. Primary care physicians in this region must be particularly trained to detect the psychiatric disorders among older adults with attention given to anxiety disorders, given their prevalence, medical and psychiatric comorbidity. Similar studies as well as epidemiological investig-

doi:10.1017/S1041610212001925

\section{Effects of vitamin B12 supplementation on cognition, depression, and fatigue in patients with lacunar stroke}

We read the review by Moore et al. (2012), recently published in this journal, with great interest and ations, are important to raise awareness toward the different types of psychiatric disorders encountered among older adults in Saudi Arabia enabling the development of more specific prevention strategies and proper management.

\section{Acknowledgment}

The authors thank Professor Brian Draper, Professor (Conjoint), School of Psychiatry, University of NSW, Sydney, Australia for his assistance in revising the manuscript

\section{Conflict of interest}

None.

\section{References}

First, M. B., Spitzer, R. L., Gibbon, M. and Williams, J. B. W. (eds.) (1997). Structured Clinical Interview for DSM-IV Axis I Disorders-Clinical Version (SCID-CV). Washington: APA Press.

Ghubash, R., El-Rufaie, O., Zoubeidi, T., Al-Shboul, Q. M. and Sabri, S. M. (2004). Profile of mental disorders among the elderly United Arab Emirates population: sociodemographic correlates. International Fournal of Geriatric Psychiatry, 19, 344-351.

Hafez, G., Bagchi, K. and Mahaini, R. (2000). Caring for the elderly: a report on the status of care for the elderly in the Eastern Mediterranean Region. Eastern Mediterranean Health Fournal, 6, 636-643.

Lyness, J. M., Caine, E. D., King, D. A., Cox, C. and Yoediono, Z. (1999). Psychiatric disorder in old primary care persons. Fournal of General Internal Medicine, 14, 249-254.

Ogunniyi, A. et al. (1998). Dementia in Saudi Arabia: experience from a university hospital. Acta Neurologica Scandinavica, 98, 116-120.

\section{Mostafa Amr, Tarek Tawfik Amin, DAHOUD AL-RHADDAD AND KHALID SALLAM}

Faculty of Medicine, Benha University, Egypt Email: sallamk2000@gmail.com compliment the authors for their thorough review of cognitive impairment and its relation to vitamin B12.

The authors identified only four intervention studies in neurological patients presenting with vitamin B12 deficiency. Although vitamin B12 is specifically associated with cerebrovascular 
pathology (Fassbender et al., 1999), none of these studies included patients with cerebrovascular pathology in particular. Additionally, the studies did not select for patients who were vitamin B12 deficient. Furthermore, cognition was assessed with the Mini-Mental State Examination only, and no assessments of depression were included (Moore et al., 2012). Recently, we found increased levels of fatigue and depression in lacunar stroke patients with vitamin B12 deficiency (Huijts et al., 2012). Possible effects of vitamin B12 supplementation on cognitive function and post-stroke fatigue and depression may significantly improve quality of life. Therefore, we performed a pilot study to determine the effect size of vitamin B12 supplementation on cognition. We included 14 first-ever lacunar stroke patients with a vitamin B12 deficiency presenting at our Neurology Department. Lacunar stroke was defined as an acute stroke syndrome with a small $(<20 \mathrm{~mm})$ ischemic lesion on acute brain magnetic resonance imaging in the brain stem, basal ganglia, or internal capsule, compatible with the occlusion of a single perforating small artery. We assessed cognitive function with an extensive neuropsychological test battery as well as fatigue (Checklist Individual Strength) (Vercoulen et al., 1999) and depressive symptoms (Hospital Anxiety and Depression Scale-Depression subscale) (Zigmond and Snaith, 1983) by self-report. Supplementation (1 $\mathrm{mg}$ hydroxocobalamine) was administered for at least three months by their general practitioner. Although preliminary, significant differences between baseline assessment and follow-up were found for verbal learning scores (Rey Auditory Verbal Learning Test (Brand and Jolles, 1985) $(p=0.008)$. This is in line with the recently published results of Walker et al. (2012). In addition, we calculated reliable change indices to determine if an individual's performance on a test has changed significantly after the intervention, while taking into account the measure's reliability (Jacobson and Truax, 1991). In line with the group analyses we found a significant improvement in verbal learning for 6 out of 14 patients (43\%). Overall, these individuals were younger $(M=56.0$ vs. $M=65.5$ years) and higher educated than those who remained stable $(n=6)$ or declined $(n=2)$, though not significant $(p=0.189$ and $p=0.068$, respectively). No effect was found for vitamin B12 supplementation on fatigue and depressive symptoms. One of the recommendations of Moore et al. (2012) was the need for studies with more robust measures of cognition. We addressed this issue, as well as the recommendation to include depression measures. Additionally, we included patients with cerebrovascular pathology who are at risk of vitamin B12 deficiency (Fassbender et al., 1999) and have not been studied before in supplementation studies. One major limitation however is the small sample size. To provide future studies with a guideline for a minimal number of patients for a randomized controlled trial, we performed sample size estimations (Erdfelder et al., 1996) based on our database. Expected differences between treatment and control group were set at $25 \%$. Taking into account a drop-out rate of $20 \%$, we estimated the minimal sample size to test a difference in memory function after supplementation to be 1,008 (504 per treatment arm). In order to detect a difference in fatigue and depression, 4,388 and 2,178 patients are needed, respectively. Assuming that $33 \%$ of lacunar stroke patients are vitamin B12 deficient (Huijts et al., 2012), 3,024, 13,164, and 6,534 lacunar stroke patients, respectively, will have to be screened in order to include these numbers. Considering these large sample sizes, future studies need a multicenter setting.

\section{Conflict of interest}

None.

\section{References}

Brand, N. and Jolles, J. (1985). Learning and retrieval rate of words presented auditorily and visually. Fournal of General Psychology, 112, 201-210.

Erdfelder, E., Faul, F. and Buchner, A. (1996). GPOWER: a general power analysis program. Behavior Research Methods, Instruments, and Computers, 28, 1-11.

Fassbender, K., Mielke, O., Bertsch, T., Nafe, B., Froschen, S. and Hennerici, M. (1999). Homocysteine in cerebral macroangiography and microangiopathy. Lancet, 353, 1586-1587.

Huijts, M., Duits, A., Staals, J. and van Oostenbrugge, R. J. (2012). Association of vitamin B12 deficiency with fatigue and depression after lacunar stroke. PLoS One, 7, e30519.

Jacobson, N. S. and Truax, P. (1991). Clinical significance: a statistical approach to defining meaningful change in psychotherapy research. Fournal of Consulting and Clinical Psychology, 59, 12-19.

Moore, E., Mander, A., Ames, D., Carne, R., Sanders, K. and Watters, D. (2012). Cognitive impairment and vitamin B12: a review. International Psychogeriatrics, 24, 541-556.

Vercoulen, J. H. M. M., Alberts, M. and Bleijenberg, G. (1999). De Checklist Individual strength (CIS). Gedragstherapie, 32, 131-136.

Walker, J. G. et al. (2012). Oral folic acid and vitamin B-12 supplementation to prevent cognitive decline in community-dwelling older adults with depressive symptoms-the Beyond Ageing Project: a randomized controlled trial. American fournal of Clinical Nutrition, 95, 194-203. 
Zigmond, A. S. and Snaith, R. P. (1983). The hospital anxiety and depression scale. Acta Psychiatrica Scandinavica, 67, 361-370.

Marjolein Huijts, ${ }^{1,2,3}$ Robert J. VAN Oostenbrugge, ${ }^{1,2,3}$ Rob P. W. Rouhl, ${ }^{1}$ Paul Menheere ${ }^{4}$ ANd ANNElien DuITs $^{3,5}$

${ }^{1}$ Department of Neurology, Maastricht University Medical Centre (MUMC), Maastricht, The
Netherlands

Email: M.Huijts@Mumc.nl

${ }^{2}$ Cardiovascular Research Institute Maastricht

(CARIM), Maastricht University, Maastricht, The Netherlands

${ }^{3}$ School for Mental Health and Neuroscience

(MHeNS), Maastricht University, Maastricht, The Netherlands

${ }^{4}$ Department of Clinical Chemistry, MUMC, Maastricht, The Netherlands

${ }^{5}$ Department of Psychiatry and Psychology, MUMC, Maastricht, The Netherlands 